\title{
PERIODONTAL DISEASE AS AN ETIOLOGY OF OROFACIAL AND MUSCULOSKELETAL PAINS IN WOMEN (Case Report)
}

\author{
Haryono Utomo," Chiquita Prahasanti." \\ "Dental Clinic, Faculty of Dentistry Airlangga University \\ "Department of Periodontology Faculty of Dentistry Airlangga University
}

\begin{abstract}
Orofacial pain includes pain associated with the hard and soft tissues of the head, face, neck, and all of the intraoral structures. Upon painful episodes, consuming analgesics or traditional medicine are relatively common. It is also a common sense that high cholesterol and hypertension may contribute to the pain. Since most pain sufferers are women, estrogen is proposed to be a modulator of pain perception. Nevertheless, the mechanism of pain modulation in women is still in controversy. Systemic manifestations of periodontal disease are widely accepted. However, the role of periodontal disease as an etiology of orofacial and musculoskeletal pains is rarely discussed. Recent study in medical psychoneuroimmunology may reveal the possibility of periodontal disease as an etiology of these painful symptoms. The objective of this study is to reveal the possibility of periodontal disease as an etiology of orofacial and musculoskeletal pains. Periodontal treatments were done to women who suffered from orofacial and musculoskeletal pains, resulting in the disappearing of the symptoms. Regarding to the amazing results, the conclusion is that especially in women, periodontal disease may cause orofacial and musculoskeletal pains especially in women. Indonesian Journal of Dentistry 2006; Edisi Khusus KPPIKG XIV:202-205
\end{abstract}

Key words: periodontal disease, orofacial pain, women

\section{Introduction}

The field of orofacial pain includes pain conditions that are associated with the hard and soft tissues of the head, face, neck, and all of the intraoral structures. The diagnostic range includes headache; musculoskeletal, neurogenic, psychogenic pains; pains from major diseases such as cancer; and sleep disorders related to orofacial pains. ${ }^{1}$

The sign and symptoms of pain in the general population have been found to occur more frequently in women than men, at ratio approximately $2: 1$. However, in populations with an age range of $15-45$, women are three to nine times more likely to suffer from painful symptoms than men. ${ }^{2}$ After the fifth decade of life, the difference is declining; it is proposed that estrogen is a possible etiology of these pain conditions. $^{2,3}$
Researches on migraine shows that women with greater vulnerability to hormonal change shows improvement after menopause. However, only $60 \%$ of women appear to be very hormonal sensitive; therefore, female sexual hormones do not seem to be the only factor influencing headache. ${ }^{4}$ There was also a study that migraine improves in $55-90 \%$ of women during pregnancy. ${ }^{3}$

The exact pathophysiological mechanism underlying the relationship between estrogen and pain remains in controversy. ${ }^{3}$ However, the possibility of periodontal disease as an etiology of painful symptoms such as orofacial and musculoskeletal pains in women is rarely discussed.

A case report by Utomo and Prahasanti had been revealed that periodontal treatments were useful in eliminating painful symptoms in women suffering from headache and dysmenorrhea. ${ }^{5}$ Nevertheless, this case report only refer to the 
late puberty women. The objective of this study is to propose periodontal disease as an etiology of orofacial and musculoskeletal pains especially in women, based on the evidence-based case reports.

\section{Case Operation Procedures}

Case 1: A 37 years old female suffered from headach, shoulder, neck and back muscles pain, especially in the right side of the body. Other symptoms were sleepy in the afternoon but sleeplessness at night. The symptoms worsen about 6 months before the treatment was done, but the symptoms had already started about 1 year earlier.

When her periods came, the pain became unbearable; she could not stand and only lay down. She had dysmenorrhea and all the pain symptoms worsen during that time. She has consulted several doctors without improvements.

At first visit, extra oral condition looked normal, except for the fatigue appearance. Intra oral examination found that there were gingival inflammation and redness in every region. Probing revealed that periodontal pockets existed in all regions and more pronounced in the posterior regions and the gingiva bleed easily.

Panoramic radiograph showed horizontal resorption in all regions, especially in lower right region where she had a splinted crowns on 4746 .

The first thing to be done was reshaping the splint bridge; widen its embrasure to favor food cleansing. Scaling was done and the reaction of scaling procedures made her felt better; headache and shoulder pain subsides instantly.

At second visit, one week later, she said that all the pain symptoms had disappeared; also she had an undisturbed sleep at night. She was also taught how to clean her splint bridge, and suggested to make a new one if the symptoms recurrent.

One month later, she was evaluated and said that the pain symptoms did not exist; and when she had her period, the symptoms related to dysmenorrhea were gone. She was evaluated in April 2006, 6 months later and the painful symptoms did not exist.

Case 2: A 47 years old female, suffered from several painful symptoms on the left side of her body such as throbbing headache, neck, shoulder, back and leg muscles pains for about 5 years. Other symptoms were dysregulation of blood pressure, heart palpitation, irritability, and nasal congestion.

She had already consulted many doctors in several countries without satisfying results. Some doctors said that she had hypertension and hormonal imbalance.
At first visit, there were gingival inflammation and redness in every region, and several fixed prosthesis. There was a cantilever bridge without rest in 37 and bridge in 252627 that caused food impaction and periodontal inflammation.

Panoramic radiograph showed a horizontal resorption in all regions, especially under the fixed prostheses of 242526 and 3736 . On 37, there was a furcation involvement and vertical resorption as well.

At that time, the treatment was initiated with elimination of retentive areas of the fixed prostheses which might cause periodontal problems especially distal of 27 and buccal of 37 which were the most retentive areas. After reshaping and scaling, the patient felt better. Patient was scheduled to have gingival curettage in one week time; chlorhexetidine $0.1 \%$ mouthwash and antibiotic (cefradoxil $500 \mathrm{mg}$ ) were prescribed. She was told to take the antibiotic 2 hours before the second visit for premedication.

At second visit, one week later, the patient reported that after the first treatment, headache and other painful symptoms in the shoulder, neck, arm and leg were disappeared. Curettage was done in 24, 26 and 37; patient was scheduled for the next visit one week later.

At third visit, the patient had no complaints; at that time she had her period and all the painful symptoms she used to suffer did not exist. The latest evaluation was in February 2006, 5 months later; and the symptoms did not recur.

Case 3: A female, aged 63, suffered from headaches, pain in the neck and shoulder, and spasm in the right hand for about one year. Especially in the thumb, index and middle finger were felt just like tied by a rope. .She had already sought for treatments by many doctors in several countries without satisfying result.

On extra oral examination at first visit, there was an asymmetry on the right side of her face. Intra orally, the gingival were inflamed red and there were a lot of calculus deposits in the lingual of the right lower posterior teeth; and also chronic periodontal abscess in 4546 .

Panoramic radiograph revealed that there was severe periodontal disease in every regions. Especially in 15 and 45, the horizontal and vertical resorptions were extensive.

At first visit when abscess still present, scaling was done carefully, especially for the abundant calculus deposits in the region of 4546 . Antibiotic, analgesic and chlorhexetidine mouthwash were prescribed; the next visit were scheduled in 5 days.

At second visit, the facial asymmetry did not exist; she also said that the headache, pain 
and muscle spasm in the neck and shoulders subsides. At that time, the scaling procedure was continued; after the treatment was finished, the patient said that the pain and spasm in her arm and fingers also felt better. Patient was scheduled for the next visit in a week time.

At third visit, all the painful symptoms, including headache and finger spasm were disappeared. The latest evaluation was in February 2006, 6 months later; the patient said that the symptoms still completely disappeared.

\section{Discussion}

Several peripheral and central mechanism through which estrogen could operate to increase pain have been postulated for orofacial pains, including enhanced inflammatory responses, actions of prostaglandin release, and receptors. ${ }^{6,7}$ Although in some situations prostaglandins contribute to pain by directly activating nociceptors, they are generally considered to be sensitizing agents. ${ }^{8}$

This case report and an article by Utomo and Prahasanti showed that, especially in women who suffered from periodontal disease, the painful symptoms (headache and musculoskeletal pains) occur from puberty until menopause. Women in these case reports who still have their menstrual period felt that the painful symptoms were worsen during their period. ${ }^{5}$

Migraine in women is attributed to abrupt withdrawal of estrogen rather than to sustain high or low estrogen levels. Abrupt withdrawal of estrogen occurs naturally in the menstrual cycle or artificially through cyclical intervention (e.g. during oral contraceptive), which causes the modulation of hypothalamic beta endorphin and the dopamine and serotonin (5-HT1 and 5-HT2) receptors. ${ }^{6,7}$

Another cause of migraine is the proinflammatory cytokines interleukin- $1 \beta$ (IL$1 \beta$ ) and nitric oxide (NO). ${ }^{9}$ It has been proposed that NO plays a crucial role in the activation of the trigeminovascular system by activating perivascular sensory afferent nerve fibers (via 5HT receptors) in the meninges. ${ }^{1,7}$

According to Nalbandian, low estrogen (17$\beta$ estradiol) as in menopause and physiological estrogen levels as in puberty till perimenopause, are stimulators of Th1 immune response that secretes proinflammatory cytokines such as IFN$\gamma$, TNF- $\alpha$ and IL-12. In the other hand, high estrogen as in pregnancy, stimulate Th2 immune response that produce antiinflammatory cytokines IL-4, IL-10 and TGF- $\beta$. $^{10}$

Regarding to the existing painful symptoms that also occurred during the luteal phase which the female sexual hormones are in their peak concentration, ${ }^{2,11}$ it seems plausible that not only female sexual hormones modulate pain perception. The role of local inflammation such as chronic periodontal disease elicited by endotoxin from gram negative bacteria (lipopolysaccharide, LPS) which may extend to systemic inflammation ${ }^{12}$ should also be considered. Lipopolysaccharide is a potent stimulator of immunocompetent cells i.e. macrophages and mast cells which release proinflammatory mediators. ${ }^{13}$

Tissue damage and inflammation as in periodontal disease produce an array of chemical mediators such as bradykinin and prostanoids. Other products are proinflammatory cytokines and neuropeptides including substance P (SP) and calcitonin gene-related peptide (CGRP) that can excite or sensitize nociceptors to elicit pain at the site of injury. ${ }^{1,14}$ Among them, prostanoids (i.e. prostaglandin $E_{2}, P_{2} E_{2}$ ) were shown to influence the inflammation. and their administration was found to reproduce the major signs of inflammation including augmented pain. $^{14}$

The inflammatory mediator $\mathrm{PGE}_{2}$ is thought to act directly on the peripheral terminals of primary afferent nociceptors via the E-prostanoid receptors. ${ }^{14}$ The effects are to produce hyperalgesia (tenderness); to sensitize nociceptors ${ }^{1,14}$, and to enhance tetrodotoxin resistant voltage-gated-sodium current (TTX-RI $\mathrm{Na}){ }^{14}$ Prostaglandin $\mathrm{E}_{2}$ and nitric oxide (NO) sensitize primary afferent neurons to bradykinin and other mediators and are likely to be involved at multiple sites along the nociceptive pathway. ${ }^{8}$

Comparing the characteristics of pain modulating substances between men and women coincidentally, basal NO serum concentration in women is higher than men. ${ }^{15}$ In addition, the anti oxidant properties of estrogen may prevents NO degradation and consequently increasing NO availability. In the presence of $\mathrm{NO}$, the state of hyperalgesia and sensitization produced by $\mathrm{PGE}_{2}$ are maintained. ${ }^{14}$

When macrophages are strongly activated, they can injure normal host tissues by the release of NO which is a non-selective microbicidal, which do not distinguish between self tissue and microbes. $^{13}$ Damaged tissue creates more prostaglandins and bradykinin, thus may induces more pain and hyperalgesia.'

Estrogen and progesterone have disadvantageous effects to gingiva, as they may cause the susceptibility of women to periodontal disease. Estrogen decreases gingival keratinization, whereas progesterone increases vasodilatation and permeability. Additionally, estrogen and progesterone may act as growth factors of Prevotella intermedia that is gram negative periodontal pathogenic bacteria. ${ }^{16}$ 
In the presence of LPS and IFN- $\gamma$, macrophages that primed with physiological level $17-\beta$ estradiol increase the production of NO. ${ }^{17}$ Interferon- $\gamma$, a Th1 cytokine which is stimulated by low or physiological estrogen level activates macrophages which then produce more proinflammatory cytokines. ${ }^{10}$

Therefore, as headache may caused by excess of IL-1, TNF- $\alpha^{9}$, and NO ${ }^{1,9}$, women with low and physiological estrogen level may suffered from headache in the presence of LPS. Coincidentally, $55-90 \%$ migraineurs improve during pregnancy, especially during the last two semesters; ${ }^{3}$ it could be related to the high estrogen level which acts as a stimulator of Th2 cytokines which is anti-inflammatory. ${ }^{10}$

Medical psychoneuroimmunology is focuses on the interactions among the Central Nervous System (CNS), the neuroendocrine system, and the immune system. The hypothalamic-pituitary-adrenal (HPA) axis is a major pathway through which immune function can be altered. ${ }^{18}$

Proinflammatory cytokines may stimulate the HPA axis which lead to the glucocorticoid (i.e. cortisol) synthesis. ${ }^{18}$ The increase of cortisol synthesis may decrease progesterone level in several ways: (1) as cortisol itself is a derivate of progesterone, increase of cortisol synthesis may lead to decrease of progesterone level ${ }^{11},(2)$ by competitive binding; as cortisol and progesterone has the same affinity both to corticoid and progesterone receptors, cortisol may binds to progesterone receptors, ${ }^{19}$ these mechanisms may reduce the circulating progesterone level.

Decrease of progesterone level may increases estrogen level and causes abrupt withdrawal of estrogen during menstrual period and the estrogen dominance. The symptoms related to estrogen dominance are headache, irregular menses, cramping in periods or midcycle pain, nasal congestion, irritability etc. ${ }^{\text {II }}$

\section{Conclusion}

Periodontal disease, which is a chronic infection; may cause orofacial and musculoskeletal pains in women at least by two ways. First, chronic infection is a source of proinflammatory mediators which stimulate the production of cortisol and decrease of progesterone level. This condition may lead to estrogen dominance and abrupt withdrawal of estrogen during the menstrual period. Second, in women with low and physiological estrogen level, LPS induced macrophages release more proinflammatory cytokines, prostaglandin $E_{2}$, nitric oxide, and bradykinin which cause painful symptoms.
As a result, in the presence of periodontal disease the state of hyperalgesia, orofacial and musculoskeletal pains may become chronic in women from puberty until menopause. Since periodontal treatments in these case reports relief these painful symptoms, it is concluded that periodontal disease has an important role in the etiology of orofacial and musculoskeletal pains in women.

\section{References}

1. Okeson JP. Bell's Orofacial Pain. $6^{\text {th }}$ ed. Carol Stream: Quintessence Publ, 2005: 6, 13

2. Gremillion HA. Multidisciplinary Diagnosis and Management of Orofacial Pain. General Dentistry 2000; 2: $178-84$

3. Bouser MG. Estrogens, Migraine, and Stroke. Stroke. 2004; 35(suppl I): 2652-6.

4. http://www.achenet.org/articles/2.php, American Council of Headache Education. Migraine. Diakses pada tanggal 12 Ooktober 2005.

5. Utomo H, Prahasanti C. Periodontal Disease in Headache and Menstrual Pain. Kumpulan Makalah Lustrum FKG Universitas Gadjah Mada. Yogyakarta: FKG UGM 2005: 101-6.

6. Gladstone JP, Eross EJ, Dodick DW. Migraine in Special Populations: Treatment Strategies for Children and Adolescent, Pregnant Women and the Elderly. Postgraduate Medicine. 2004: 115(4).

7. Fettes I. Menstrual Migraine: Methods of Prevention and Control. Postgraduate medicine 1997; 101(5) Available online at URL http://www. postgradmed.com. Accessed Oct 12 2005.

8. Kidd BL, Urband LA. Mechanisms of Inflammatory Pain. Brit J Anaesth 2001; 87(1): 3-11.

9. Marteletti P. Inflammatory Mechanisms in Cervicogenic Headache: An Integrative view. Current Science 2002; 6: $315-9$.

10. Nalbandian G, Kovats S. Estrogen, Immunity and Autoimmune Disease. Curr Med Chem-Immun Endoc Metab Agents 2005; 5: 85-91.

11. http://www.lammd.com, Lam M. Estrogen Dominance: the Silent Epidemic, Diakses pada tanggal 12 November 2005

12. Li XJ, Kolltveit KM, Tronstad L. Systemic Diseases Caused by Oral Infection. Clin Microb Rev 2000; 13(4):547-58

13. Abbas AK, Lichtman AH. Cellular and Molecular Immunology. $5^{\text {th }}$ ed.. Philadelphia: WB Saunders, 2002: 288.

14. Moriyama T, Higashi T, Togashi $\mathrm{K}$. Sensitization of TRPV1 by EP1 and IP Reveals Peripheral Nociceptive Mechanism of Prostaglandins. Molecular Pain 2005; 1(3): 1-13.

15. Tostes RC, Nigro D, Fortes ZB. Effects of Estrogen on the Vascular System. Braz J Med Biol Res 2003; 36: 1143-58.

16. Güncü $\mathrm{CN}$, Tözüm $\mathrm{TF}$, Cağlayan $\mathrm{F}$. Effects of Endogenous Sex Hormones on the Periodontium. Austr Dent J 2005; 50(3): $138-45$.

17. Sakazaki H, Ido R, Ueno H. 17ק Estradiol Primes Elicitation of Inducible Nitric Oxide Synthase Expression by Lipopolysaccharide and Interferon- $\gamma$ in Mouse Macrophage Cell Line J774.1. J Health Sci 2005; 51(1): 62-9.

18. Padgett DA, Glaser R. How Stress Influences the Immune Response. Trends in Immunology 2003; 24(8): 444-8.

19. Quinckler M, Meyer B, Burnke-Vogt C. Agonistic and Antagonistic Properties of Progesterone Metabolites at the Human Mineralocorticoid Receptor. Eur $J$ Endocrinol 2002; 146: 789-800. 